\title{
An Analysis of Vertical Splitting Algorithm
}

\author{
Ruchika Bhaskar \\ Research Student \\ Computer Science Department \\ Rajasthan Technical University Jaipur, Rajasthan, \\ India
}

\author{
Rakesh Sharma \\ Associate Professor \\ Computer Science Department \\ Rajasthan Technical University, Jaipur, Rajasthan, \\ India
}

\begin{abstract}
Distribution design involves making decisions on the fragmentation and allocation of data across the sites of a computer network. Vertical splitting is the process of subdividing the attributes of a relation to generate fragments. In this paper, we propose an analysis for vertical splitting algorithm using prototype approach. This approach starts from the attribute affinity matrix and generates initial clusters based on the affinity values between attributes. Then, it uses the database according to optimal splitting solution to produce final groups that will represent the fragments. Then we analysed these fragments according to their contribution level. The result is generated that shows how to find optimal solutions.
\end{abstract}

\section{Keywords}

BEA, Distributed Database, Vertical Fragmentation.

\section{INTRODUCTION}

Distributed and parallel processing on database management systems (DBMS) is an efficient way of enhancing performance of applications that work on large volumes of data. This may be accomplished by eliminating irrelevant data accessed during the execution of queries and by reducing the data exchange among sites, which are the two main goals of the design of distributed databases [2].

The primary concern of distributed database systems is to design the fragmentation and allocation of the underlying database. The designing of distribution involves making decisions on the fragmentation and placement of data across the sites of a computer network. The first phase of the distribution design in a top-down approach is the fragmentation phase, which is the process of clustering into fragments the data accessed simultaneously by applications. The fragmentation phase is then followed by the allocation phase, which handles the physical storage of the generated fragments among the nodes of a computer network, and the replication of fragments.

\section{RELATED WORK}

Most of the vertical splitting algorithms have started from constructing an attribute affinity matrix from the attribute usage matrix: the Attribute affinity matrix is an $\mathrm{m} \mathrm{x} \mathrm{m} \mathrm{matrix}$ for the m-attribute problem whose $(i, j)$ element equals the "between attributes" affinity which is the total number of accesses of transactions referencing both attributes $i$ and $j$. An iterative binary partitioning method has been used in [8] and [5] based on first clustering the attributes and then applying empirical objective functions or mathematical cost functions to perform the fragmentation. The concept of using fragmentation of data as a means of improving the performance of a database management system has often appeared in the literature on file design and optimization. Attribute partitioning and attribute clustering have been studied earlier by [4], [3], [6], [8], [9] has discussed the implementation of a self-reorganizing database management system that carries out attribute clustering. They also show that in a database management system where storage cost is low compared to the cost of accessing the sub files, it is beneficial to cluster the attributes, since the increase in storage cost will be more than offset by the saving in access cost. Hoffer [6] developed a non-linear, zero-one program, which minimizes a linear combination of storage, retrieval and update costs, with capacity constraints for each file.

Navathe et al [8] used a two-step approach for vertical partitioning. In the first step, they used the given input parameters in the form of an attribute usage matrix to construct the attribute affinity matrix on which clustering is performed. After clustering, an empirical objective function is used to perform iterative binary partitioning. In the second step, estimated cost factors reflecting the physical environment of fragment storage are considered for further refinement of the partitioning scheme. Cornell and $\mathrm{Yu}$ [5] proposed an algorithm, as an extension of Navathe et al [8] approach, which decreases the number of disk accesses to obtain an optimal binary partitioning. This algorithm uses specific physical factors such as number of attributes, their length and selectivity, cardinality of the relation etc.

Navathe and Ra have developed a new algorithm based on a graphical technique [7]. This algorithm starts from the attribute affinity matrix by considering it as a complete graph called the "affinity graph" in which an edge value represents the affinity 1-4244-1364-8/07/\$25.00 @2007 IEEE between the two attributes, and then forms a linearly connected spanning tree. The algorithm generates all meaningful fragments in single iteration by considering a cycle as a fragment. A linearly connected tree has only two ends. By a "linearly connected tree" we imply a tree that is constructed by including one edge at a time such that only edges at the "first" and the "last" node of the tree would be considered for inclusion. We then form "affinity cycles" in this spanning tree by including the edges of high affinity value around the nodes and "growing" these cycles as large as possible. After the cycles are formed, partitions are easily generated by cutting the cycles apart along "cut-edges". In this paper we will use an algorithm to cluster the database i.e. Bond Energy Algorithm (BEA). And use these cluster affinity as input to find final fragments using PARTITION algorithm. Then using prototypes we reach to the goal of reducing response time of query using fragmentation and show the mathematical result for proof.

\section{BACKGROUND OF SPLITTING ALGORITHM}

Today, mostly centralized databases are used to store and manage data [11]. They carry the advantages of high degree of security, concurrency, backup and recovery control. However, they also have the disadvantages of high communication costs 
(when the client is far away and communication is very frequent in between the client \& server), unavailability in case of system failure and a single source bottleneck [3].

Research conducted in 1991 for distributed databases predicted a huge shift from traditional databases to distributed databases in the coming arena, due to organizational needs to manage huge amounts of data [11]

The telecommunication sector also wants to embrace this technology of data distribution. But before distribution of data, fragmentation is a very important and critical task that needs to be done.

Most of the telecom industries are using centralized technique in storage of their database. Centralized database has its disadvantage of high communication cost. Some data is unavailable due to problem in server. To resolve these issues we are moving from centralized database to distribution of the database.

\section{ANALYSIS OF ALGORITHM USED FOR SPLITTING THE DATABASE}

The vertical fragmentation proposed in this paper is executed in following manner. (1) When a query uses attribute from a relation its value is true (2) Information about databases and query are notified before fragmentation process. (3)Query consists of attributes. The use of attribute means accessing to the value of an existing attribute without any side-effect. Our fragmentation is composed of attribute fragmentation. A set of attributes defined in a relation is vertically partitioned into attribute fragments on basis of application queries like

\author{
1. Normal \\ Find subscriber via peer_id and area \\ Find Narrative \\ 2. Recharge \\ Find subscriber via peer_id and area \\ Find subscriber_id \\ Find Narrative(units) \\ Update Narrative(units) \\ Update subscriber (Sub_Param1, 2, 3) \\ 3. Balance Inquiry \\ Find subscriber via peer_id and area \\ Find Narrartives etc.
}

\section{Figure 1: Sample Queries of experiment}

After, the attribute fragments are generated, the attribute fragmentation is executed on basis of queries .Also, a query can access attributes of other relation on a database hierarchy. To reflect these characteristics of queries, we calculate QA(query access) matrix, FA(site access) matrix and AU(attribute usage) matrix. In FA matrix, QA matrix contains all explanation in of attribute used by Sites of different relations and to represent attributes usage by different queries.

\subsection{QA matrix:}

QA (Query Access) matrix represents the use of attributes in application queries. Figure 2 and Figure 3 is an example of the QA matrix for subscriber relation and Narrative relation used as database in this paper.
11110111010
11010000000
11011000000
1111000000000
111010000000
11010000100
111000000000
1010000000000
10100000000

\section{Figure 2: SUBSCR QA Matrix}
111111
111110
111010
1111100
00000000
00000000
0111110
00000000
010100

\section{Figure 3: NARRATIVE QA Matrix}

Each row represents queries and each column represents attributes Q1-A1, Q1-A2, Q1-A3.....Q1-A11 are queries about SUBSCR relation. And in the similar manner for NARRATIVE relation (in Figure 3). The entry "1" indicates that the query uses the corresponding attributes. Attributes of other relation accessed by query can be represented in QA matrix. For example, Q1 accesses to not only sub_id, peer_id, status, language of its own relation SUBSCR but also narrative_id, sub_id, narrative_type, and unit_type of relation NARRATIVE.

$$
\begin{aligned}
& \text { Combined AQ matrix (CAQ) } \\
& 1111011101011111 \\
& 1101000000011110 \\
& 1101100000011010 \\
& 1111000000011100 \\
& 1110100000000000 \\
& 1101000010000000 \\
& 1110000000001110 \\
& 1010000000000000 \\
& 1010000000000100
\end{aligned}
$$

Figure 4: Combined AQ matrix (CAQ)

\subsection{FA Matrix:}

The access frequency represents the sum of number of accesses about query generated in one or more sites.

Figure 5 shows the access frequency of different queries by different sites In QA matrix, A usage value of attribute $\mathrm{Aj}$ for a query qi is defined as:

$\mathbf{u s e}(\mathbf{q i}, \mathbf{A} \mathbf{j})=\mathbf{1}$ if query qi accesses to

$$
\mathbf{A j}=\mathbf{0} \text { otherwise }
$$




$\begin{array}{llll} & \text { S1 } & \text { S2 } & \text { S3 } \\ \text { Q1 } & 10 & 15 & 5 \\ \text { Q2 } & 0 & 3 & 2 \\ \text { Q3 } & 15 & 1 & 0 \\ \text { Q4 } & 0 & 5 & 6 \\ \text { Q5 } & 8 & 0 & 0 \\ \text { Q6 } & 25 & 20 & 25 \\ \text { Q7 } & 0 & 0 & 10 \\ \text { Q8 } & 0 & 6 & 4 \\ \text { Q9 } & 2 & 8 & 20\end{array}$

Figure 5: Access Frequency Matrix(FA)

The AA (attribute affinity) matrix is generated from the AQ matrix using the same technique as relational vertical fragmentation approach.

Attribute Affinity Matrix aff(Ai, Aj)

The attribute affinity represents the strength of bond between the two attributes. The attribute affinity for two attributes Ai and $\mathrm{Aj}$ defined as

$\operatorname{aff}(A i, A j)=k \mid$ use $(q k, A i)=1^{\wedge}$ use $(q k, A j)=1$ acc $(q k)$

where $\operatorname{aff}(\mathrm{Ai}, \mathrm{Aj})$ is affinity value between $\mathrm{Ai}$ and $\mathrm{Aj}, \operatorname{acc}(\mathrm{qk})$ is the total number of access of query qk generated in multiple sites. Figure 6 and Figure 7 shows an example of AA matrix.

The AA matrix is needed to be clustered, and then becomes to divide into attribute fragments. Bond Energy Algorithm (BEA) is used to cluster the AA matrix. The algorithm is as following:

\section{Algorithm: BEA}

Input: AA attribute affinity matrix

Output: CA clustered affinity matrix

Begin

\{Initialize; remember that $\mathrm{AA}$ is an $\mathrm{n} \times \mathrm{n}$ Matrix

$\mathrm{CA}(\mathrm{R} \cdot, 1) \leftarrow \mathrm{AA}(\mathrm{R}, 1)$

$\mathrm{CA}(\mathrm{R}, 2) \leftarrow \mathrm{AA}(\mathrm{R}, 2)$

index $\leftarrow 3$

while index $\leq \mathrm{n}$ do \{choose the "best" location for attribute AAindex $\}$

begin

for I from 1 to index-1 by 1 do

calculate cont(Aindex-1, Aindex, Aindex+1)

end-for

calculate cont(Aindex-1, Aindex, Aindex+1) \{boundary condition \}

location $\leftarrow$ placement given by maximum cont value

for $\mathrm{j}$ from index to location by -1 do $\quad$ shuffle the two matrices\}

$\mathrm{CA}(\mathrm{R}, \mathrm{j}) \leftarrow \mathrm{AA}(\mathrm{R}, \mathrm{j}-1)$

end-for

$\mathrm{CA}(\mathrm{R}$, location $) \leftarrow \mathrm{AA}(\mathrm{R}$,index $)$

index $\leftarrow$ index +1

end-while

order the rows according to the relative ordering of columns end. $\{$ BEA $\}$

The purpose of clustering is to combine large affinity value of AA matrix with large affinity values, and the small one with small ones.

\section{IMPLEMENTATION AND COMPARISION}

We have implemented the vertical class fragmentation proposed in this paper using JAVA programming language on an IBM-PC. The implementation was executed in the following procedures.

First, establish the example structure of class schema and example queries with the access frequency.

Second, generate the QA, AU matrix for all tables.

Third, for each table, generate AA and CA matrixes.

Fourth, partition the CA matrixes and make the attribute fragments.

When we apply both BEA algorithm [16] and vertical Partition algorithm [16] according to the Attribute usage matrix and Attribute Affinity matrix we conclude to the fragment results and selected one of the optimal solutions available.

\subsection{Testing Results}

In this section we analysed the result. After applying algorithm we find following result.

\begin{tabular}{|c|c|}
\hline Query & Sum of Attribute access \\
\hline q1: & 30 \\
\hline q2: & 5 \\
\hline q3: & 16 \\
\hline q4: & 11 \\
\hline q5: & 8 \\
\hline q6: & 70 \\
\hline q7: & 10 \\
\hline q8: & 10 \\
\hline q9: & 30 \\
\hline
\end{tabular}

Figure 6: Query Total Access Frequency

q1: A1 A10 A11 A2 A3 A4 A6 A7 A8 30

q2: A1 A2 A9 5

q3: A1 A2 A9 16

q4: A1 A2 A3 A9 11

q5: A1 A2 A3 A9 8

q6: A1 A2 A8 A9 70

q7: A1 A2 A3 10

q8: A1 A3 10

q9: A1 A3 30

Figure 7: Attribute Usage with frequency

A1 A10 A11 A2 A3 A4 A6 A7 A8 A9 A1 $1903030150 \quad 993030 \quad 30100110$ $\begin{array}{lllllllllll}\mathrm{A} 10 & 30 & 30 & 30 & 30 & 30 & 30 & 30 & 30 & 30 & 0\end{array}$ $\begin{array}{llllllllllll}\mathrm{A} 11 & 30 & 30 & 30 & 30 & 30 & 30 & 30 & 30 & 30 & 0\end{array}$ A2 $150 \quad 30 \quad 30150 \quad 5930 \quad 30 \quad 30100110$

A3 $\quad 99 \begin{array}{lllllllll}30 & 30 & 59 & 99 & 30 & 30 & 30 & 30 & 19\end{array}$

A4 $30 \begin{array}{lllllllll}30 & 30 & 30 & 30 & 30 & 30 & 30 & 30 & 0\end{array}$

A6 $\begin{array}{lllllllllll}30 & 30 & 30 & 30 & 30 & 30 & 30 & 30 & 30 & 0\end{array}$

A7 $30 \quad 30 \quad 30 \quad 30 \quad 30 \quad 30 \quad 30 \quad 30 \quad 30 \quad 0$

A8 $100 \quad 30 \quad 30100 \quad 30 \quad 30 \quad 30 \quad 30100 \quad 70$

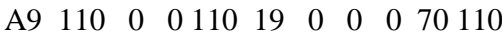

\section{Figure 8: Attribute Affinity Matrix Subscr}

In Figure 8, A10 comes after A1 it depends upon the bond energy which can be calculated for individual column randomly.

Calculate the contribution of the column depending on its position: 


\subsubsection{Place attributes:}

\section{place $\mathrm{A} 1$}

contribution at pos $0=20670$

contribution at pos $1=85841$

contribution at pos $2=83441$

attribute A1 is placed at pos 1: [A4, A1, A2]

\section{place A10}

contribution at pos $0=8100$

contribution at pos $1=8100$

contribution at pos $2=-44501$

contribution at pos $3=18270$

attribute A10 is placed at pos 3: [A4, A1, A2, A10]

\section{place A11}

contribution at pos $0=8100$

contribution at pos $1=8100$

contribution at pos $2=-44501$

contribution at pos $3=8100$

contribution at pos $4=8100$

attribute A11 is placed at pos 0: [A11, A4, A1, A2, A10]

place A3

contribution at pos $0=13110$

contribution at pos $1=18120$

contribution at pos $2=39491$

contribution at pos $3=2741$

contribution at pos $4=33971$

contribution at pos $5=13110$

attribute $\mathrm{A} 3$ is placed at pos 2: [A11, A4, A3, A1, A2, A10]

place A6

contribution at pos $0=8100$

contribution at pos $1=8100$

contribution at pos $2=8100$

contribution at pos $3=-13271$

contribution at pos $4=-44501$

contribution at pos $5=8100$

contribution at pos $6=8100$

attribute A6 is placed at pos 0: [A6, A11, A4, A3, A1, A2, A10]

place A7

contribution at pos $0=8100$

contribution at pos $1=8100$

contribution at pos $2=8100$

contribution at pos $3=8100$

contribution at pos $4=-13271$

contribution at pos $5=-44501$

contribution at pos $6=8100$

contribution at pos $7=8100$

attribute A7 is placed at pos 0: [A7, A6, A11, A4, A3, A1, $\mathrm{A} 2, \mathrm{~A} 10]$

place A8

contribution at pos $0=14400$

contribution at pos $1=20700$

contribution at pos $2=20700$

contribution at pos $3=20700$

contribution at pos $4=28890$

contribution at pos $5=39719$

contribution at pos $6=29699$

contribution at pos $7=50100$

contribution at pos $8=14400$

attribute $\mathrm{A} 8$ is placed at pos 7: [A7, A6, A11, A4, A3, A1, A2, A8, A10] place A9

contribution at pos $0=9270$

contribution at pos $1=10440$

contribution at pos $2=10440$

contribution at pos $3=10440$

contribution at pos $4=19611$

contribution at pos $5=34781$

contribution at pos $6=28161$

contribution at pos $7=36521$

contribution at $\operatorname{pos} 8=32140$

contribution at pos $9=9270$

attribute A9 is placed at pos 7: [A7, A6, A11, A4, A3, A1, A2, A9, A8, A10]

The order below leads to the highest bond energy.

resulting order: [A7, A6, A11, A4, A3, A1, A2, A9, A8, A10]

There are now several possibilities to split the table:

split quality $(\mathrm{sq})=\operatorname{accesses}($ fragment 1$) * \operatorname{accesses}($ fragment 2) - accesses(fragment 1 AND fragment 2)^2

\subsubsection{Find fragments:}

split at [A1, A10, A11, A2, A3, A4, A7, A8, A9] | [A6]

accesses frag 1 alone: 160

accesses frag2 alone: 0

accesses frag1 and frag2: 30

split quality $=-900$

split at [A1, A10, A2, A3, A4, A7, A8, A9] | [A11, A6]

accesses frag1 alone: 160

accesses frag 2 alone: 0

accesses frag1 and frag2: 30

split quality $=-900$

split at [A1, A10, A2, A3, A7, A8, A9] | [A11, A4, A6]

accesses frag 1 alone: 160

accesses frag2 alone: 0

accesses frag1 and frag2: 30

split quality $=-900$

split at [A1, A10, A2, A7, A8, A9] | [A11, A3, A4, A6] accesses frag1 alone: 91

accesses frag 2 alone: 0

accesses frag1 and frag2: 99

split quality $=-9801$

split at [A10, A2, A7, A8, A9] | [A1, A11, A3, A4, A6]

accesses frag 1 alone: 0

accesses frag 2 alone: 40

accesses frag1 and frag2: 150

split quality $=-22500$

split at [A10, A7, A8, A9] | [A1, A11, A2, A3, A4, A6] accesses frag 1 alone: 0

accesses frag2 alone: 50

accesses frag1 and frag2: 140

split quality $=-19600$

split at [A10, A7, A8] | [A1, A11, A2, A3, A4, A6, A9]

accesses frag1 alone: 0

accesses frag2 alone: 90

accesses frag1 and frag2: 100

split quality $=-10000$

split at [A10, A7] | [A1, A11, A2, A3, A4, A6, A8, A9] accesses frag1 alone: 0 
accesses frag2 alone: 160

accesses frag1 and frag2: 30

split quality $=-900$

split at [A7] | [A1, A10, A11, A2, A3, A4, A6, A8, A9] accesses frag1 alone: 0

accesses frag2 alone: 160

accesses frag1 and frag2: 30

split quality $=-900$

split at [A1, A10, A2, A3, A4, A6, A7, A8, A9] | [A11]

accesses frag1 alone: 160

accesses frag2 alone: 0

accesses frag1 and frag2: 30

split quality $=-900$

split at [A1, A10, A2, A3, A6, A7, A8, A9] |[A11, A4] accesses frag1 alone: 160

accesses frag2 alone: 0

accesses frag1 and frag2: 30

split quality $=-900$

split at [A1, A10, A2, A6, A7, A8, A9] | [A11, A3, A4]

accesses frag1 alone: 91

accesses frag 2 alone: 0

accesses frag1 and frag2: 99

split quality $=-9801$

split at [A10, A2, A6, A7, A8, A9] | [A1, A11, A3, A4] accesses frag1 alone: 0

accesses frag2 alone: 40

accesses frag1 and frag2: 150

split quality $=-22500$

split at [A10, A6, A7, A8, A9] |[A1, A11, A2, A3, A4]

accesses frag1 alone: 0

accesses frag 2 alone: 50

accesses frag1 and frag2: 140

split quality $=-19600$

split at [A10, A6, A7, A8] | [A1, A11, A2, A3, A4, A9] accesses frag 1 alone: 0

accesses frag2 alone: 90

accesses frag1 and frag2: 100

split quality $=-10000$

split at [A10, A6, A7] | [A1, A11, A2, A3, A4, A8, A9]

accesses frag1 alone: 0

accesses frag2 alone: 160

accesses frag1 and frag2: 30

split quality $=-900$

split at [A6, A7] | [A1, A10, A11, A2, A3, A4, A8, A9]

accesses frag 1 alone: 0

accesses frag2 alone: 160

accesses frag1 and frag2: 30

split quality $=-900$

split at [A1, A10, A11, A2, A3, A6, A7, A8, A9] | [A4] accesses frag1 alone: 160

accesses frag 2 alone: 0

accesses frag1 and frag2: 30

split quality $=-900$

split at [A1, A10, A11, A2, A6, A7, A8, A9] |[A3, A4]

accesses frag 1 alone: 91

accesses frag 2 alone: 0 accesses frag1 and frag2: 99

split quality $=-9801$

split at [A10, A11, A2, A6, A7, A8, A9] | [A1, A3, A4]

accesses frag1 alone: 0

accesses frag 2 alone: 40

accesses frag1 and frag2: 150

split quality $=-22500$

split at [A10, A11, A6, A7, A8, A9] | [A1, A2, A3, A4]

accesses frag 1 alone: 0

accesses frag2 alone: 50

accesses frag1 and frag2: 140

split quality $=-19600$

split at [A10, A11, A6, A7, A8]|[A1, A2, A3, A4, A9]

accesses frag1 alone: 0

accesses frag2 alone: 90

accesses frag1 and frag2: 100

split quality $=-10000$

split at [A10, A11, A6, A7] | [A1, A2, A3, A4, A8, A9] accesses frag1 alone: 0

accesses frag2 alone: 160

accesses frag1 and frag2: 30

split quality $=-900$

split at [A11, A6, A7] |[A1, A10, A2, A3, A4, A8, A9] accesses frag 1 alone: 0

accesses frag2 alone: 160

accesses frag1 and frag2: 30

split quality $=-900$

split at [A1, A10, A11, A2, A4, A6, A7, A8, A9] | [A3]

accesses frag1 alone: 91

accesses frag 2 alone: 0

accesses frag1 and frag2: 99

split quality $=-9801$

split at [A10, A11, A2, A4, A6, A7, A8, A9] | [A1, A3]

accesses frag1 alone: 0

accesses frag 2 alone: 40

accesses frag1 and frag2: 150

split quality $=-22500$

split at [A10, A11, A4, A6, A7, A8, A9] | [A1, A2, A3]

accesses frag 1 alone: 0

accesses frag2 alone: 50

accesses frag1 and frag2: 140

split quality $=-19600$

split at [A10, A11, A4, A6, A7, A8] | [A1, A2, A3, A9]

accesses frag 1 alone: 0

accesses frag2 alone: 90

accesses frag1 and frag2: 100

split quality $=-10000$

split at [A10, A11, A4, A6, A7] | [A1, A2, A3, A8, A9]

accesses frag1 alone: 0

accesses frag2 alone: 160

accesses frag1 and frag2: 30

split quality $=-900$

split at [A11, A4, A6, A7] | [A1, A10, A2, A3, A8, A9]

accesses frag 1 alone: 0

accesses frag 2 alone: 160

accesses frag1 and frag2: 30 
split quality $=-900$

split at [A10, A11, A2, A3, A4, A6, A7, A8, A9] | [A1] accesses frag 1 alone: 0

accesses frag2 alone: 0

accesses frag1 and frag2: 190

split quality $=-36100$

split at [A10, A11, A3, A4, A6, A7, A8, A9] |[A1, A2]

accesses frag 1 alone: 0

accesses frag 2 alone: 0

accesses frag1 and frag2: 190

split quality $=-36100$

split at [A10, A11, A3, A4, A6, A7, A8] | [A1, A2, A9] accesses frag1 alone: 0

accesses frag2 alone: 21

accesses frag1 and frag2: 169

split quality $=-28561$

split at [A10, A11, A3, A4, A6, A7] | [A1, A2, A8, A9]

accesses frag1 alone: 0

accesses frag 2 alone: 91

accesses frag1 and frag2: 99

split quality $=-9801$

split at [A11, A3, A4, A6, A7] | [A1, A10, A2, A8, A9] accesses frag1 alone: 0

accesses frag2 alone: 9

accesses frag1 and frag2: 99

split quality $=-9801$

split at [A1, A10, A11, A3, A4, A6, A7, A8, A9] | [A2]

accesses frag 1 alone: 40

accesses frag2 alone: 0

accesses frag1 and frag2: 150

split quality $=-22500$

split at [A1, A10, A11, A3, A4, A6, A7, A8] |[A2, A9]

accesses frag 1 alone: 40

accesses frag2 alone: 0

accesses frag1 and frag2: 150

split quality $=-22500$

split at [A1, A10, A11, A3, A4, A6, A7] | [A2, A8, A9] accesses frag1 alone: 40

accesses frag 2 alone: 0

accesses frag 1 and frag2: 150

split quality $=-22500$

split at [A1, A11, A3, A4, A6, A7] |[A10, A2, A8, A9]

accesses frag1 alone: 40

accesses frag 2 alone: 0

accesses frag1 and frag2: 150

split quality $=-22500$

split at [A1, A10, A11, A2, A3, A4, A6, A7, A8] | [A9] accesses frag1 alone: 80

accesses frag 2 alone: 0

accesses frag1 and frag2: 110

split quality $=-12100$

split at [A1, A10, A11, A2, A3, A4, A6, A7] | [A8, A9]

accesses frag1 alone: 50

accesses frag 2 alone: 0

accesses frag1 and frag2: 140

split quality $=-19600$ split at [A1, A11, A2, A3, A4, A6, A7] | [A10, A8, A9]

accesses frag1 alone: 50

accesses frag 2 alone: 0

accesses frag1 and frag2: 140

split quality $=-19600$

split at [A1, A10, A11, A2, A3, A4, A6, A7, A9] |[A8]

accesses frag1 alone: 90

accesses frag2 alone: 0

accesses frag1 and frag2: 100

split quality $=-10000$

split at [A1, A11, A2, A3, A4, A6, A7, A9] |[A10, A8]

accesses frag1 alone: 90

accesses frag2 alone: 0

accesses frag1 and frag2: 100

split quality $=-10000$

split at [A1, A11, A2, A3, A4, A6, A7, A8, A9] | [A10]

accesses frag 1 alone: 160

accesses frag 2 alone: 0

accesses frag1 and frag2: 30

split quality $=-900$

optimal split(s) $(\mathbf{s q}=\mathbf{- 9 0 0})$ :

[A1, A10, A11, A2, A3, A4, A7, A8, A9] | [A6]

[A1, A10, A2, A3, A4, A7, A8, A9] | [A11, A6]

[A1, A10, A2, A3, A7, A8, A9] |[A11, A4, A6]

[A10, A7] | [A1, A11, A2, A3, A4, A6, A8, A9]

[A7]|[A1, A10, A11, A2, A3, A4, A6, A8, A9]

[A1, A10, A2, A3, A4, A6, A7, A8, A9] |[A11]

[A1, A10, A2, A3, A6, A7, A8, A9] |[A11, A4]

[A10, A6, A7] |[A1, A11, A2, A3, A4, A8, A9]

[A6, A7] |[A1, A10, A11, A2, A3, A4, A8, A9]

[A1, A10, A11, A2, A3, A6, A7, A8, A9] |[A4]

[A10, A11, A6, A7] | [A1, A2, A3, A4, A8, A9]

[A11, A6, A7]|[A1, A10, A2, A3, A4, A8, A9]

[A10, A11, A4, A6, A7] |[A1, A2, A3, A8, A9]

$[\mathrm{A} 11, \mathrm{~A} 4, \mathrm{~A} 6, \mathrm{~A} 7] \mid[\mathrm{A} 1, \mathrm{~A} 10, \mathrm{~A} 2, \mathrm{~A} 3, \mathrm{~A} 8, \mathrm{~A} 9]$

[A1, A11, A2, A3, A4, A6, A7, A8, A9] |[A10]

Similar approach can be used for NARRATIVE relation and we find the following solution. The following order leads to highest bond energy

resulting order: $[\mathrm{A} 6, \mathrm{~A} 5, \mathrm{~A} 3, \mathrm{~A} 2, \mathrm{~A} 4, \mathrm{~A} 1]$

and optimal split(s) (sq = 13180):

[A6] | [A1, A2, A3, A4, A5]

So following fragments has been selected with primary key [16].

Fragments of Subscr

[A1,A11, A6, A7] |[A1, A10, A2, A3, A4, A8, A9]

Fragments of Narrative

[A1,A6] | [A1, A2, A3, A4, A5]

\section{CONCLUSION}

The purpose of conducting this study is to know the comparative study of various vertical splitting techniques. We know in centralized databases horizontal fragmentation is widely used because of its ease of methods available. But vertical fragmentation is rarely used to do the fragments .so our study presents a view for ease of use of vertical fragmentation. 
Distributed databases have many aspects and every organization has certain preferences. For the telecom sector, the response time is prioritized.

Our experiment showed that the how to calculate the fragments for different criteria used. In the distributed database, data is fragmented. These fragments are short compared to the full database (centralized database contains maximum columns). However, when we need data from multiple sites for a query (report queries), the response time is increased. Accessing data from multiple remote sites and then joining those takes long time. But in the centralized database since data is at one place so, it is easy and fast to search it. The purpose of conducting this study is to know the distributed databases using vertical fragmentation. How to do attribute distribution in vertical fragmentation?

Answer: This question is depending upon the data available and algorithms available. Anyone can choose its data according to heuristic approach. Based on algorithms available for vertical fragmentation both type of databases, and experiment was performed. Experiment results are discussed in section 5 .We found that the response time is decreased in distributed databases. Because, due to fragmentation data set for single site contains less records than centralized database, so response time is less.

\section{REFERENCES}

[1] Ceri, S. and Pelagatti, G. Distributed DatabasesPrinciples and Systems. NY, McGraw Hill, 1984.

[2] Ezeife, C. I. and Barker, K. Vertical Class Fragmentation in a Distributed Object Based Svstem. TR 94-03, Univ. of Manitoba DeRt. ofCbmputer Science, 1993.

[3] H.o ffer. 1. A.. and Severance. D. G. The Use of Cluster Analysis in Physical Database Design.In Proceedings of 1st VLDB Conference, Mass., 1975.

[4] Karlapalem, K. and Li, 8. Partitioning Schemes for Object Oriented Database. In 5th InternationalWorkshop on Research Issues on Data Engineering: Distributed Object Management, 1995.

[5] Karlapalem, K., Li, 8. and Vieweg, S. Method Induced Partitioning Schemes in Object OrientedDatabases. In 16th intemational conference on Distributed Computing System, Hong Kong, 1996.

[6] Karlapalem, K., Navathe, S. B. and Morsi, M. M.A. Issues in Distribution design. of object-oriented databases, in Distributed Object Management, Morgan Kaufmann Publishers, 1994.

[7] Lee, S. and Lim, H., Extension of Vertical Technical Conference on Circuits/systems, Computers And Communications, Japan, 1997.

[8] Navathe, S. B., Ceri, S. Wiederhold, G. and Dou, J.Vertical partitioning algorithms for database design.in ACM TODS 9(4), 1984.

[9] Farhi Marir, Yahiya Najjar, Mahmoud Y. AlFaress, Hassan I. Abdalla, "An Enhanced Grouping Algorithm for Vertical Partitioning Problem in DDBs

[10] Adrian Runceanu, Towards Vertical Fragmentation in Distributed Databases

[11] Ashraf, Imran And Khokhar, A.S. 2010. Principles for Distributed Databases in Telecom Environment., Sweden.

[12] Huang, Y.-F. And Chen, J.-H. 2001. Fragment Allocation in Distributed Database Design.

[13] Mitchell , C. Components of a Distributed Database.

[14] Jonker, W. 2000. Databases in telecommunications: international workshop co-located with VLDB-99, Edinburgh, Scotland, UK, September 6th 1999: proceedings. Springer, Berlin.

[15] Hvasshovd, S.-O. 1995. the clustRa telecom database: high availability high throughput and real-time response Proceedings of 21st VLDB Conference.

[16] Wiederhold, G., and Dou, J.,"Vertical Partitioning Algorithms for Database Design," ACM Trans.on Database Systems, Vol. 9, No.4, Dec. 1984. 